\title{
Estudo do anel mitral e trígonos fibrosos com diferentes variáveis
}

\author{
Fábio B. JATENE*, Rosângela MONTEIRO*, Marcelo B. JATENE*, Maria H. G. MAGALHĀES*, Júlia
} T. FUKUSHIMA*, Adib D. JATENE*

JATENE, F. B.; MONTEIRO, R.; JATENE, M. B.; MAGALHÃES, M. H. G.; FUKUSHIMA, J. T.; JATENE, A. D. - Estudo do anel mitral e trígonos fibrosos com diferentes variáveis. Rev. Bras. Cir. Cardiovasc., 6(3):190-194, 1991.

RESUMO: O objetivo do presente estudo é avaliar dados anatốmicos importantes na cirurgia reconstrutora da valva mitral, em particular características do anel mitral (AM) relacionadas a posiçāo e distância dos trígonos fibrosos, Além disso, buscamos correlacionar esse aspecto com as variáveis sexo, idade, grupo étnico e posiçāo do anel aórtico em relação ao AM - "posiçāo mitro-aórtica". Foram estudados 96 coraçōes humanos previamente fixados, nos quais foram realizadas duas medidas no $A M$, sendo a medida $A$ a maior distância entre os trígonos fibrosos e a B, a menor distância entre os mesmos, resultando a soma das duas medidas no perímetro total do anel. Dividindo-se os valores de $A$ pelos valores de $B$, estabeleceuse a relação $A / B$, que variou de 1,88 a 3,96 $(2,69+0,38)$. $A$ análise desta relação não apresentou variação estatística quando correlacionada individualmente com sexo $(p=0,6857)$ e idade $(p=0,1157)$. Porém, quando feita associação das variáveis grupo étnico e "posiçāo mitro-aórtica", observou-se que, em coraçōes de indivíduos nāo brancos, houve diminuiçāo da relação $A / B$ quando a posição "mitro-aórtiça" era anterior e muito anterior $(p=0,0285)$. Em conclusão, a relaçäo $A / B$ não é fixa, apresentando variaçöes na dependência da associação dos fatores grupo étnico e "posição mitro-aórtica".

DESCRITORES: valva mitral, anatomia.

\section{INTRODUÇÃO}

Várias doenças afetam as valvas cardíacas, produzindo lesões anatômicas que comprometem o seu funcionamento. A valva mitral é uma das mais acometidas pela doença², que afeta não só suas cúspides, mas principalmente o anel fibroso que freqüentemente apresenta diâmetro anormal ${ }^{5}$.

O emprego de técnicas cirúrgicas conservadoras na valva mitral vem sendo cada vez mais freqüente, em função dos bons resultados obtidos ${ }^{3,16,17}$. Isso é reforçado pelos inúmeros problemas que acompanham a colocação de próteses como roturas, calcificações, deterioração mecânica e complicações tromboembólicas ${ }^{8,10,11,14,21-23}$.

O conhecimento mais apurado da anatomia assume, portanto, importante papel no sucesso das plastias valvares, já que muitas decisōes tomadas durante a cirurgia são baseadas na avaliação visual das anormalidades ${ }^{12}$. Além disso, a reconstrução valvar, além de resultado funcional satisfatório, deve proporcionar retorno à normalidade anatômica ${ }^{6}$.

Em estudo prévio, foi observado que os coraçōes apresentam diferentes relaçōes entre suas principais estruturas, particularmente a relação entre os anéis mitral e aórtico, denominada "posição mitroaórtica"15.

O objetivo do presente estudo é avaliar dados anatômicos importantes na cirurgia reconstrutora da valva mitral, em particular características do anel mitral (AM) relacionadas a posição e distância dos trígonos fibrosos. Além de buscar associação desse

Trabalho realizado no Museu Anatômico Cirúrgico do Instituto do Coração do Hospital das Clínicas da Faculdade de Medicina da Universidade de São Paulo. São Paulo, SP, Brasil.

Recebido para publicação em 4 de novembro, 1991.

*Do Instituto do Coração do Hospital das Clínicas da Faculdade de Medicina da Universidade de São Paulo.

Endereço para separatas: Fábio B. Jatene. Av. Dr. Enéas de Carvalho Aguiar, 44. Divisão Cirúrgica. 05403 São Paulo, SP, Brasil. 
JATENE, F. B.; MONTEIRO, R.; JATENE, M. B.; MAGALHĀES, M. H. G.; FUKUSHIMA, J. T.; JATENE, A. D. - Estudo do anel mitral e trígonos fibrosos com diferentes variáveis. Rev. Bras. Cir. Cardiovasc., 6(3):190-194, 1991.

aspecto com as variáveis sexo, idade, grupo étnico e "posição mitro-aórtica".

\section{MATERIAL E MÉTODOS}

Foram estudados 96 corações humanos considerados anatomicamente normais, obtidos de cadáveres sem cardiopatia prévia, sendo sua "causa mortis" o traumatismo agudo. Quanto a grupo étnico, $36(37,5 \%)$ eram de indivíduos não-brancos. Oitenta $(83,3 \%)$ pertenciam a indivíduos do sexo masculino, com idades entre 9 e 86 anos $(30,95+15,37)$. Quanto a posição do anel aórtico em relação ao AM - "posição mitro-aórtica" - 47 (48,96\%) tinham posição média, $27(28,12 \%)$ muito anterior e $22(22,92 \%)$ anterior.

Os coraçōes estudados tiveram os átrios rebatidos para possibilitar a visibilização da base do coração. Com o auxílio de fio de algodão zero e de um paquímetro, foram feitas duas medidas no $A M$, sendo a medida A a maior distância entre os trígonos fibrosos e a medida $B$, a menor distância. O resultado da soma das duas medidas representava o perímetro total do AM (Figura 1). Estabeleceu-se a relação $A / B$, dividindo-se os valores de $A$ pelos valores de $B$.

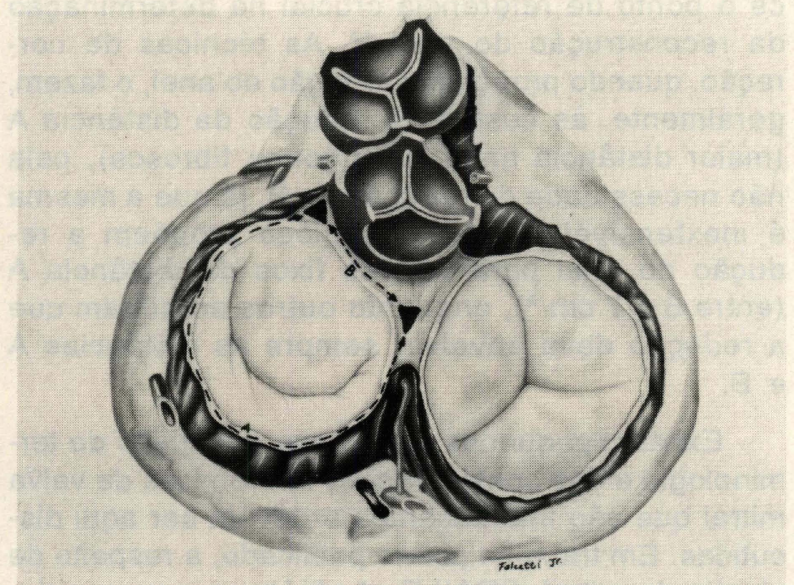

Fig. 1 - Esquema da base do coração representando as valvas cardíacas e os trígonos fibrosos anterior e posterior. $A=$ maior distância entre os trígonos fibrosos; $\mathrm{B}=$ menor distância entre os trígonos fibrosos.

\section{Análise Estatística}

$A$ relação $A / B$ foi avaliada através do ajuste de um modelo linear geral, onde foram considerados os fatores sexo, grupo étnico, "posição mitro-aórtica", a variável idade (em valores absolutos) e as interaçōes de primeira ordem entre todas as variáveis independentes. Os efeitos de interação e os efeitos princi- pais foram testados através da soma de quadrados tipo $1 \mathrm{II}^{20}$.

\section{RESULTADOS}

A relação $A / B$ variou de 1,88 a $3,96(2,69+$ $0,38)$. A análise do modelo linear geral revelou não haver efeito de interação estatisticamente significativo quando correlacionadas as variáveis sexo e grupo étnico; sexo e "posição mitro-aórtica"; idade e sexo; grupo étnico e idade; idade e "posição mitroaórtica". A mesma análise do modelo linear geral revelou haver efeito de interação quando correlacionadas as variáveis grupo étnico e "posição mitroaórtica" ( $p=0,0285)$ (Tabela 1). Em função disso, as variáveis sexo e idade foram analisadas isoladamente e as variáveis grupo étnico e "posição mitroaórtica" foram analisadas conjuntamente.

TABELA 1

RESULTADOS DA ANÁLISE DE VARIÂNCIA - VARIÁVEL DEPENDENTE: RELAÇĀO AVB

\begin{tabular}{|c|c|c|c|c|}
\hline & & $\begin{array}{l}\text { Fonte de } \\
\text { variação }\end{array}$ & $\begin{array}{c}\text { Estatística } \\
\mathrm{F}\end{array}$ & $p^{*}$ \\
\hline $\begin{array}{l}\text { Efeitos } \\
\text { Principais }\end{array}$ & $\begin{array}{l}\text { Sexo } \\
\text { Idade }\end{array}$ & & $\begin{array}{l}0,16 \\
2,53\end{array}$ & $\begin{array}{l}0,6857 \\
0,1157\end{array}$ \\
\hline $\begin{array}{l}\text { Efeitos } \\
\text { Interaçäo }\end{array}$ & $\begin{array}{l}\text { Sexo X } \\
\text { Sexo X } \\
\text { Idade X } \\
\text { Gr étnico X } \\
\text { Gr étnico X } \\
\text { Idade X }\end{array}$ & $\begin{array}{l}\text { Gr étnico } \\
\text { Posição MA } \\
\text { Sexo } \\
\text { Posição MA } \\
\text { Idade } \\
\text { Posição MA }\end{array}$ & $\begin{array}{l}0,01 \\
0,87 \\
0,43 \\
3,72 \\
0,06 \\
0,37\end{array}$ & $\begin{array}{l}0,9828 \\
0,4235 \\
0,5147 \\
0,0285 \\
0,8053 \\
0,6903\end{array}$ \\
\hline
\end{tabular}

* p: probabilidade de significância $; \mathrm{Gr}=$ grupo; $M A=$ mitro-aórtica

A análise das variáveis sexo e idade não apresentou associação com a relação $A / B$, sendo, respectivamente, $p=0,6857$ e $p=0,1157$ (Tabela 2).

TABELA 2

VALORES MÍNIMOS E MÁXIMOS, MÉDIAS, DESVIOS PADRÓES DA VARIÁVEL A/B, POR SEXO

\begin{tabular}{lcccc}
\hline Sexo & Mínimo & Máximo & Média & $\begin{array}{l}\text { Desvio } \\
\text { Padrão }\end{array}$ \\
\hline Feminino $(16,67 \%)$ & 1,88 & 3,14 & 2,69 & 0,35 \\
Masculino (83,33\%) & 2,05 & 3,96 & 2,68 & 0,39 \\
\hline
\end{tabular}

$p=0,6857$ 
JATENE, F. B.; MONTEIRO, R.; JATENE, M. B.; MAGALHÄES, M. H. G.; FUKUSHIMA, J. T.; JATENE, A. D. - Estudo do anel mitral e trígonos fibrosos com diferentes variáveis. Rev. Bras. Cir. Cardiovasc., 6(3):190-194, 1991.

Com relação à análise conjunta das variáveis grupo étnico e "posição mitro-aórtica", verificamos que, em coraçōes de indivíduos não-brancos, quanto mais anterior foi a posição, menor foi a média observada da relação $A / B$, o mesmo não se observando no grupo étnico branco (Tabela 3 ).

TABELA 3

VALORES MÍNIMOS E MÁXIMOS, MÉDIAS, DESVIOS PADRÕES DA VARIÁVEL AVB, POR GRUPO ÉTNICO E "POSIÇĀO MITRO-AÓRTICA"

\begin{tabular}{llllll}
\hline $\begin{array}{l}\text { Grupo } \\
\text { étnico }\end{array}$ & $\begin{array}{c}\text { Posição } \\
\text { MA }\end{array}$ & Mínimo & Máximo & Média & $\begin{array}{c}\text { Desvio } \\
\text { Padrão }\end{array}$ \\
\hline Branca & Média & 1,88 & 3,21 & 2,59 & 0,30 \\
& Anterior & 2,30 & 2,96 & 2,54 & 0,19 \\
& Mt. Anterior & 2,06 & 3,20 & 2,83 & 0,31 \\
Não & Média & 2,19 & 3,96 & 2,90 & 0,60 \\
Branca & Anterior & 2,09 & 3,12 & 2,74 & 0,33 \\
& Mt. Anterior & 2,05 & 3,31 & 2,57 & 0,40 \\
\hline
\end{tabular}

$\mathrm{ML}=$ Muito; $\mathrm{MA}=$ mitro-aórtica .

$p=0,0285$

\section{COMENTÁRIOS}

O conceito clássico de que 0 anel mitral é uma estrutura circular bem definida, situada ao redor do orifício atrioventricular esquerdo vem sendo reformulado nos últimos anos. Reconhece-se, hoje, uma continuidade anatômica entre diversas estruturas fibrosas encontradas na via de entrada do VE, todas agrupadas sob a denominaçāo genérica de membrana aortoventricular ${ }^{9}$. O anel fibroso, na sua porção anterior, faz parte do esqueleto fibroso do coração estando diretamente relacionado aos trígonos fibrosos esquerdo e direito. Assim, o termo anel usado neste estudo refere-se a uma estrutura que está longe de ser circular completa perfeita ${ }^{12,24}$.

Os métodos cirúrgicos conservadores para correção, além da abordagem sobre alteraçōes de cúspides, cordoalha e aparelho subvalvar, geralmente englobam formas de redução do anel freqüentemente dilatado, às posiçōes originais ${ }^{3,6,18}$.

Isso pode ser muito bem observado na literatura, onde inúmeros trabalhos relatam experiências para essa redução ou readaptação do anel, freqüentemente com o emprego de próteses específicas ${ }^{3,7,13,19}$. Uma das mais conhecidas e utilizadas é a prótese introduzida por CARPENTIER et alii ${ }^{3.4}$, que busca a redução do anel mitral, aplicando-se muito bem aos casos onde há um aumento, sobretudo do diâmetro ântero-posterior. Desta forma, embora a cirurgia de plastia da mitral implique na abordagem das várias estruturas da valva, fica claro que a ação sobre o anel valvar repre-

\section{GRÁFICO 1 \\ DISTRIBUIÇÃO DAS FREQÜÊNCIAS DOS CORAÇŌES ANALISADOS POR FAIXA ETÁRIA E CLASSES DA RELAÇÄO AVB}

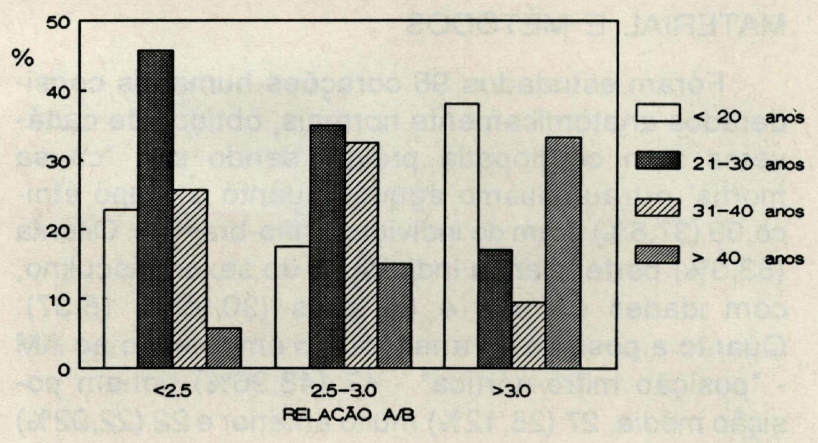

senta um passo praticamente obrigatório neste tipo de operação. Este estudo ilustra a necessidade de se conhecer a anatomia do anel mitral e sua relação com os trígonos fibrosos em situaçōes normais, para que se possa buscar a readaptação deste anel, em situações de dilatação.

Existe o conceito de que a menor distância entre os trígonos fibrosos, chamada por nós de $B$, seria inextensível por estar colocada entre os trígonos fibrosos e sobre uma estrutura fibrosa, o corpo fibruso central ${ }^{12,25}$. Esse detalhe anatômico fornece o ponto de referência crucial na determinação da reconstrução do anel ${ }^{12}$. As técnicas de correção, quando propōem a redução do anel, o fazem, geralmente, às custas da redução da distância $A$ (maior distância entre os trígonos fibrosos), pela não necessidade de redução de $B$, já que a mesma é inextensível. Alguns cirurgiōes propōem a redução do anel para valores fixos da distância $A$ (entre 6 e $7 \mathrm{~cm} *$ ), enquanto outros acreditam que a redução deva envolver sempre as distâncias $A$ e $B$.

Existem pequenas diferenças a respeito da terminologia e das considerações anatômicas da valva mitral que são interessantes e devem ser aqui discutidas. Em trabalho prévio publicado, a respeito de plastia da mitral, BRAILE et alii ${ }^{1}$ buscaram a redução da porção posterior do anel mitral com utilização de prótese maleável, fixada cirurgicamente por pontos ao anel. $O$ anel mitral seria constituído por duas porçōes: porção anterior, referente à cuspide anterior e à posterior, que, após a redução, deveria retornar ao tamanho normal, correspondente à cúspide posterior. As duas porçōes seriam delimitadas pelo ponto correspondente às comissuras e aos trígonos, que seriam, no anel, os limites aos pontos cirúrgicos passados na porção posterior. O tamanho

* JATENE, A. D. - Comunicação pessoal, 1990. 
JATENE, F. B.; MONTEIRO, R.; JATENE, M. B.; MAGALHÄES, M. H. G.; FUKUSHIMA, J. T.; JATENE, A. D. - Estudo do anel mitral e trígonos fibrosos com diferentes variáveis. Rev. Bras. Cir. Cardiovasc., 6(3):190-194, 1991.

da prótese seria correspondente ao perímetro da cúspide anterior. Na nossa observação, os trígonos nāo foram coincidentes com as comissuras e estäo colocados em um ponto tal que a menor distância entre os mesmos seria mais reduzida que o perímetro correspondente à cúspide anterior. Isto pode ser comprovado pelos nossos resultados, que demonstraram que a maior distância entre os trígonos (que englobaria a cúspide posterior e parte da cúspide anterior) foi de 1,88 a 3,96 vezes a menor distância entre os mesmos, denominada por nós de distância $B$. Essas pequenas diferenças conceituais a respeito da anatomia não impedem a obtenção de bons resultados cirúrgicos como relatado por Braile. Entretanto, é importante que sejam bem analisadas e compreendidas.
As variaçōes da relação $A / B$ não estiveram na dependência das variáveis sexo e idade. A única variaçāo estatisticamente significativa foi a correlação simultânea da relação $A / B$ com grupo étnico e "posição mitro-aórtica". O que se observou nesse caso é que, no grupo étnico não-branco, houve uma redução progressiva da média da relação $A / B$ quanto mais anterior foi a posição.

Em conclusāo, a análise, em coraçōes normais, no anel mitral, da relação entre as distâncias $A$ e B (respectivamente, a maior e a menor distância entre os trígonos fibrosos) não apresentou associação com sexo e idade. Entretanto, houve associação dessa relação quando foram analisadas conjuntamente as variáveis grupo étnico e "posição mitro-aórtica".

\section{RBCCV 44205-150}

JATENE, F.B.; MONTEIRO, R.; JATENE, M.B.; MAGALHÄES, M.H.G.; FUKUSHIMA, J.T.; JATENE, A.D. - Study of mitral ring and fibrous, trigone with different variables. Rev. Bras. Cir. Cardiovasc., 6(3):190194, 1991

ABSTRACT - The purpose of this study is to evaluate important anatomic data in the mitral valve reconstruction, specially mitral ring characteristics related to position and distance of fibrous trigone. We seek correlation of this aspect with sex, age, ethnic group and "mitral-aortic position". Ninety-four human normal hearts were analysed. Two measures were realized in mitral ring, the measure $A$ the higher distance between the fibrous trigone and $B$ the smaller distance: the sum of the two measures represent total perimeter of the ring. We established the relation A/B that changed 1.88 to 3.96 (2.69 $+0.38)$. The analysis of this relation don't present statistical difference when correlated individually with sex $(p=0.6857)$, age $(p=0.1157)$. However, in the association of the variables ethnic group and "mitral-aortic position" ( $p=0.0285)$, we observed that in hearts from non white persons was tendency to decrease of the relation $A / B$ in anterior and much anterior position. In conclusion, the relation $A /$ $B$ is not fixed, presented variation in dependence of association by ethnic group and "mitral-aortic position".

DESCRIPTORS: heart valves, mitral, anatomy.

\section{REFERÊNCIAS BIBLIOGRÁFICAS}

1 BRAILE, D. M.; ARDITO, R. V.; PINTO, G. H.; SANTOS, J. L. V.; ZAIANTCHICK, M.; SOUZA, D. R. S.; THEVENARD, R. - Plástica mitral. Rev. Bras. Cir. cardiovasc., 5:86-98, 1990.

BRAUNWALD, E. - Heart disease: a textbook of cardiovascular medicine. 4. ed. Philadelphia, W. B. Saunders Company, 1992. p. 1027-1028.

CARPENTIER, A. - Cardiac valve surgery: the "french correction". J. Thorac. Cardiovasc. Surg., 86: 322 $337,1983$. new reconstructive operation for correction of mitral and tricuspid insufficiency. J. Thorac. Cardiovasc. Surg., 61:1-13, 1971.

5 DURAN, C. M. G. - Acquired disease of mitral valve. In: BAUE, A. E.; GEHA, A. S.; HAMMOND, G. L.; LAKS, H.; NAUNHEIM, K. S. - Glenn's thoracic and cardiovascular sugery. 5. ed. Califórnia. Appleton \& Lange, 1991. Vol II, p. 1677-1696.

6 DURAN, C. M. G. - Thechniques in mitral valve reconstruction. In: STAREK, P. J. 'K. Heart valve replacement and reconstruction: clinical issues and trends. Chicago, Year Book Medical Publishers Inc., 1987. p. 115-123. 
JATENE, F. B.; MONTEIRO, R.; JATENE, M. B.; MAGALHÄES, M. H. G.; FUKUSHIMA, J. T.; JATENE, A. D. - Estudo do anel mitral e trígonos fibrosos com diferentes variáveis. Rev. Bras. Cir. Cardiovasc., 6(3):190-194, 1991.

7 DURAN, C. M. G \& UBAGE, J. M. L. - Clinical and hemodynamic performance of a totally flexible prosthetic ring for atrioventricular valve reconstruction. Ann. Thorac. Surg., 22:458-465, 1976.

8 FONTES, R. D.; POMERANTZEFF, P. M. A.; GRINBERG, M.; SNITCOWSKY, R.; VERGINELLI, G.; ZERBINI. E. J.; JATENE, A.D. - Calcificação de bioprótese de dura-máter: estudo em pacientes jovens. Rev. Paul. Med., 103: 235-238, 1985.

9 FORTUNA, A. B. P. - Anatomia cirúrgica da estenose mitral: aspecto valvar. Campinas, 1983 (Tese. Livre docência. Faculdade de Medicina da Universidade de Campinas).

FRANKL, W. S. - The special problems of the patient with a valvular prosthesis. In: FRANKL, W. S.; BREST, A. N. Valvular heart disease: comprehensive evaluation and management. Philadelphia, F. A. Davis Company, 1986. p. 415-426.

11 GALLOWAY, A. C.; COLVIN, S. B. L; BAUMANN, F. G.; GROSSI, E. A.; RIBAKOVE, G. H.; HARTY, S.; SPENCER, F. C. - A comparison of mitral valve reconstruction with mitral valve replacement: intermediate-term results. Ann. Thorac. Surg., 47: 655-662, 1989.

GALLOWAY, A. C.; COLVIN, S. B.; BAUMANN, F. G.; HARTY. S.; SPENCER, F. C. - Current concepts of mitral valve reconstruction for mitral insufficiency. Circulation, 78: 1087-1098, 1988.

13. GREGORI Jr. F.; SILVA S. S.; BABA, K.; QUEIROZ, L. T.; TAKEDA, R.; FAÇANHA, L. A.; SHIGUERU, S.; CANESIN, O. - Um novo modelo de anel protético para pacientes com insuficiência valvar mitral: relato de dois casos. Arq. Bras. Cardiol., 50: 417-420, 1988.

IONESCU, M. I.; TANDON, A. P.; CHIDAMBARAM, M.; YAKIREVICH, V. S.; SILVERTON, M. P. - Durability of the pericardial valve. Eur. Heart J., 85 (Supl. D): 101-106, 1984.
MAGALHÄES, M. H. G.; POMERANTZEFF, P. M.; JATENE, A. D. - Estudo anatômico do posicionamento do anel aórtico em relação ao anel mitral. Arq. Bras. Cardiol., 57: 203-205, 1991.

KIRKLIN, J. W. - Mitral valve repair for mitral incompetence. Mod. Concep. Cardiovasc. Dis., 56: 7-11, 1987.

17 KIRKLIN, J. W. \& BARRATT-BOYES, B. G. - Cardiac surgery: morphology, diagnostic criteria, natural history, techniques, results, and indications. New york. John Wiley \& Sons, Inc., 1986. p. 364.

18 NUNLEY, D. L. \& STARR, A. - The evoiution of reparative techniques for the mitral valve. Ann. Thorac. Surg. 37:393-397, 1984.

19 PERIER, P.; DELOCHE, A.; CHAUVAUD, S.; FABIANI, J. N.; - Comparative evaluation of mitral valve repair and replacement with Starr, Björk, and porcine valve prostheses. Circulation, 70 (Supl. 1): 187-192, 1984.

20 RAO, C. R. - Linear statistical inference and its applications. New York, Jonh Wiley \& Sons, Inc., 1965.

21 ROBERTS, W. C. - Complications of cardiac valve replacement: characteristic abnormalities of prostheses pertaining to any or specific site. Am. Heart J., 103: 113-122, 1982.

22 SAND, M. E.; NAFTEL, D. C.; BLACKSTONE, E. H.; KIRKLIN, J. W.; KARP, R. B. - A comparison of repair and replacement for mitral valve incompetence. J. Thorac. Cardiovasc. Surg., 94: 208-219, 1987.

23 SCHOEN, F. J. - Cardiac valve prostheses: pathological and bioengineering consideration. J. Cardiac Surg., 2: $65-108,1987$.

24 WALMSLEY, R. - Anatomy of human mitral valve in adult cadaver and comparative anatomy of the valve Br. Heart J., 40:351-366, 1978.

25 WILCOX, B. R. \& ANDERSON, R. M. - Surgical anatomy of the heart. London, Gower Medical Publishing, 1985. p. 2.16-2.18. 